\title{
A comparison of mass parameters determination using capacitive and optical sensors
}

\author{
V. Carvalho ${ }^{\mathrm{a}, *}$, M. Belsley ${ }^{\mathrm{b}}$, R. Vasconcelos ${ }^{\mathrm{c}}$, F. Soares ${ }^{\mathrm{a}}$ \\ a Dept. Indus. Electronics, Minho University, 4800058 Guimarães, Portugal \\ b Dept. Physics, Minho University, 4710 057, Braga, Portugal \\ ${ }^{\mathrm{c}}$ Dept. Textile Engineering. Minho University, 4800058 Guimarães, Portugal
}

\section{A R T I C L E I N F O}

\section{Article history:}

Received 13 October 2010

Received in revised form 28 February 2011

Accepted 1 March 2011

Available online 8 March 2011

\section{Keywords:}

Yarn mass parameterization

Diameter and mass correlation

Optical sensors

Capacitive sensors

Optical signal processing

\begin{abstract}
A B S T R A C T
This paper presents a comparison study between the use of $1 \mathrm{~mm}$ resolution capacitive and optical sensors for the determination of yarn mass parameters. A parallel plate capacitive sensor to determine yarn mass variations and a yarn diameter and hairiness determination solution using optical sensors and integrating optical signal processing based on Fourier analysis are described. As there is a high correlation between yarn diameter and yarn mass, it is possible to determine yarn mass and infer variations in yarn diameter and vice versa. Moreover, by optically detecting the degree of yarn hairiness, one can quantify its influence on the capacitive sensor mass variation measurements. Here we present the results of a signal processing analysis and statistical description of measurements carried out in two $100 \%$ cotton yarns with a linear mass of 59 and $295 \mathrm{~g} / \mathrm{km}$ performed in yarn system quality (YSQ) machine. We conclude that an accurate yarn characterization can be carried out using optical sensors alone, reducing system's cost and complexity and increasing efficiency.
\end{abstract}

(c) 2011 Elsevier B.V. All rights reserved.

\section{Introduction}

Mass parameters are important elements of yarn quality assessment. These include yarn diameter, mass and hairiness. To measure yarn mass, electronic capacitance testers are usually applied as a convenient and reliable method (determination of linear mass). The most commonly used industrial systems, such as ZT5 (Zweigle) and Tester 5 (Uster) use capacitors with $8 \mathrm{~mm}$ length, allowing measurements with $8 \mathrm{~mm}$ resolution. However, as most of the irregularities (Fig. 1) have a shorter length, an assessment evaluated in $1 \mathrm{~mm}$ range is of utmost importance for a correct and direct characterization [1].

There are three kinds of yarn faults, classified as shown in Fig. 1: thin places - a decrease in the mass during a short length $(4 \mathrm{~mm})$; thick places - an increase in the mass, usually lower than $100 \%$ of sensitivity, and lasting more than $4 \mathrm{~mm}$; neps - huge amount of yarn mass (equal or superior to $100 \%$ of sensitivity) in a short length (typically from $1 \mathrm{~mm}$ to $4 \mathrm{~mm}$ ) [2-5]. Sensitivity is defined as the yarn mass value used to detect a particular fault and is expressed in terms of the average yarn mass [2]. As an example, $60 \%$ of sensitivity to classify thick places means that mass measurements above 0.4 of the mass average are considered a fault; for thin places, below 0.6 of the mass average.

\footnotetext{
* Corresponding author.

E-mail address: vcarvalho@dei.uminho.pt (V. Carvalho).
}

Optical sensors are also used to measure yarn diameter and hairiness directly (Fig. 2). Hairiness is the result of released fibres over the strand. Fig. 2 presents an example of hairiness [5,6]. In addition, optical sensors also allow an indirect measurement of yarn mass due to the relationship between linear mass and yarn diameter, as can be observed in Fig. 3.

The most commonly used commercial system based on this methodology is the Oasys from Zweigle, which employs a sample measurement field of $2 \mathrm{~mm}$. Also, in this system, yarn hairiness can have a significant and undesired influence by reducing the signal received by the optical sensor, consequently leading to diameter measurement with an error by excess [7]. To measure hairiness, the traditional equipment ZT5 (Zweigle) and Tester 5 (Uster) use a dark field optics based technique. However, this method has several drawbacks that can cause a significant measurement error, namely the inexistence of a $0 \%$ of hairiness reference, the consideration of the yarn contours, the dependence on the yarn shape and alignment and without a proportional signal output to the quantity of hairiness $[8,9]$.

The present work is aimed at carrying out tests with $1 \mathrm{~mm}$ samples using capacitive and optical sensors to compare yarn mass parameters results obtained in the YSQ equipment.

\section{The measurement hardware}

This section describes the yarn mass, diameter and hairiness measurement hardware implemented in YSQ machine (Fig. 4). 


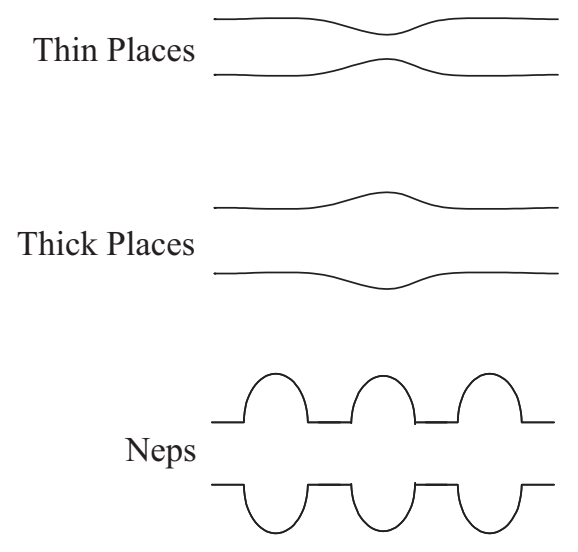

Fig. 1. Types of yarn irregularities.

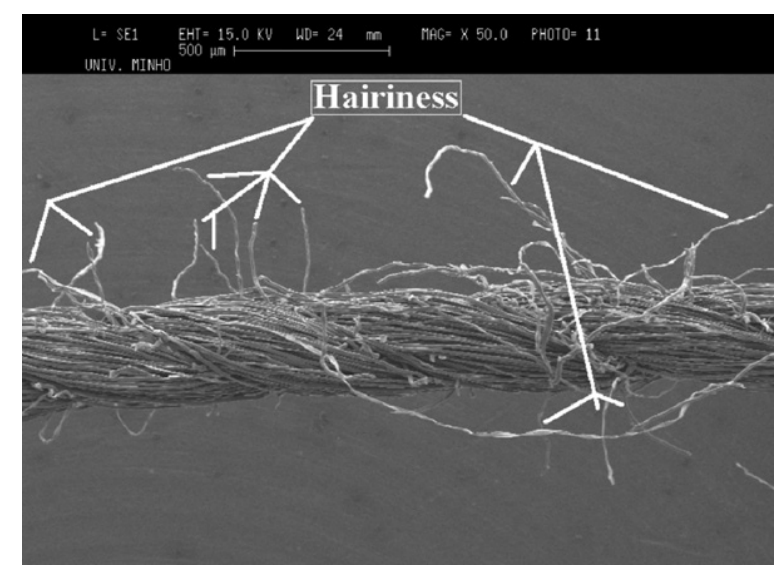

Fig. 2. Identification of yarn hairiness in an electron microscope photograph.

These characteristics of the measurement hardware allow a superior yarn parameterization, high resolution and precision [1].

\subsection{Yarn mass variation system}

The yarn mass variation system employed uses a $1 \mathrm{~mm}$ parallel plate capacitive sensor based on the integrated circuit MS3110 from Irvine Sensors, allowing direct yarn mass measurements over sample lengths of $1 \mathrm{~mm}$. The sensor adopts a differential configuration to assure a higher robustness to variations in temperature, air humidity and pressure. It integrates transducer amplification and signal conditioning, as shown in Fig. 5 [1].

As shown in Fig. 5 the sensor capacitance variation is converted into a voltage signal and amplified. A second order low-pass filter attenuates the high frequency interferences that come from an internal oscillator and other external noise sources. The filtered signal is then once more amplified.

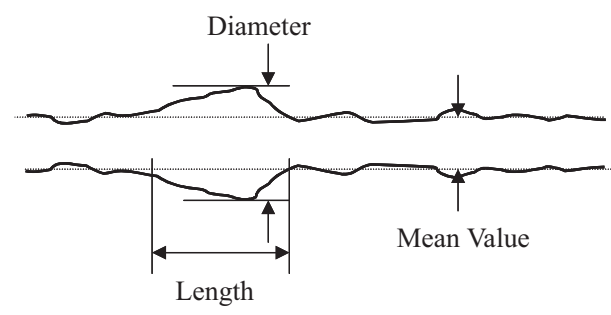

Fig. 3. Yarn configuration example.

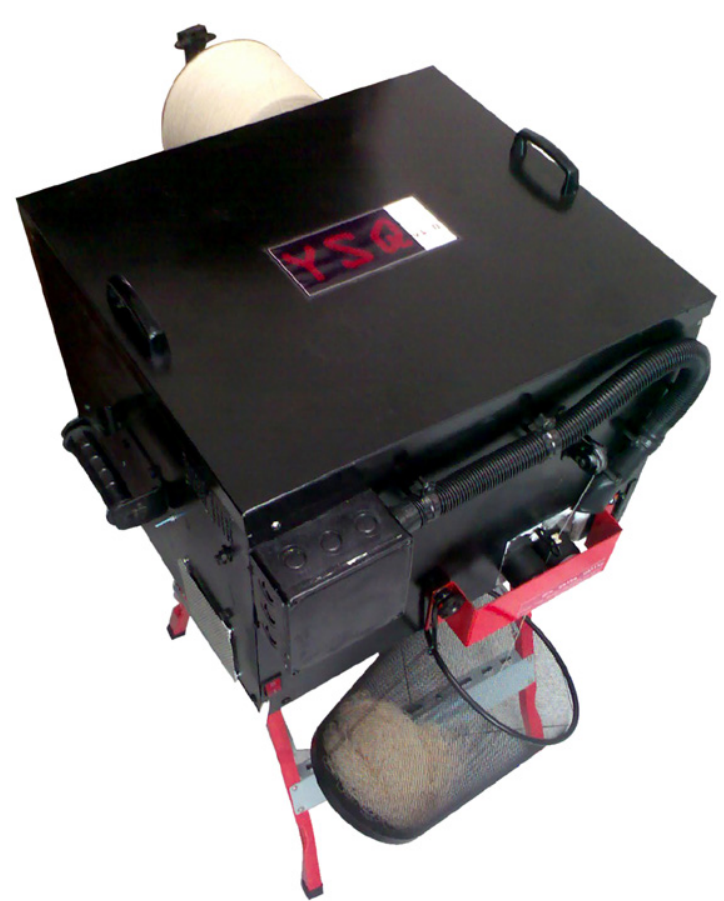

Fig. 4. YSQ prototype.

\subsection{Yarn diameter and hairiness quantification}

The yarn diameter and hairiness quantification, based on two single photodiodes (S1227-1010BR from Hamamatsu) configured for a $1 \mathrm{~mm}$ sample length analysis, uses an optical setup with a low-pass spatial filter, to perform the diameter measurement (eliminating the influence of hairiness, Fig. 6a), as well as with a high-pass spatial filter, to perform the hairiness measurement (eliminating the influence of the light which is not blocked by the yarn, Fig. 6b) [1,7-9].

Fig. 7 presents the optical hardware employed to determine yarn diameter and hairiness. A coherent optical imaging technique is employed to obtain an optical signal proportional to the amount of hairiness present on the yarn being sampled.

Adiode laser (Eudyna FLD6A2TK) was used as an illumination source. This laser emits light at $685 \pm 10 \mathrm{~nm}$ in both a single transverse and single longitudinal mode, with an especially low aspect ratio of 1.3. Emitting in a single longitudinal mode, the coherence length of the laser light is expected to be greater than several metres. The light from this laser was collimated using a single plano-convex lens of $40 \mathrm{~mm}$ focal length (L1). After illuminating the sample under study $(0)$, the light passes through a $60 \mathrm{~mm}$ focal length, $50 \mathrm{~mm}$ diameter plano-convex lens (L2) used to obtain the spatial Fourier transform of the object in its principal focal plane. A roughly $1 \mathrm{~mm}$ diameter opaque target $(F)$ placed in the Fourier plane, blocks all spatial frequencies below $10 \mathrm{~mm}^{-1}$, corresponding to a characteristic size of $100 \mu \mathrm{m}$ or larger in the object plane. The filter is chosen to pass all of the relevant information regarding the small fibres, while almost entirely eliminating the nearly constant background light due to laser light that is not incident on the sample. However, the amount of light transmitted by the spatial filter is relatively small, roughly 3 to 4 orders of magnitude less than the full power emitted by the laser. A third plano-convex lens of $60 \mathrm{~mm}$ focal length (L3) is used to form a final filtered image (position of the photo detector in Fig. 4) (I/PD), which then highlights the sharp transitions in the object, basically the core boundary of the fibre and any small protruding hairs from the yarn core, allowing the measurement of hairiness. All lenses are 1 inch in diameter, except 


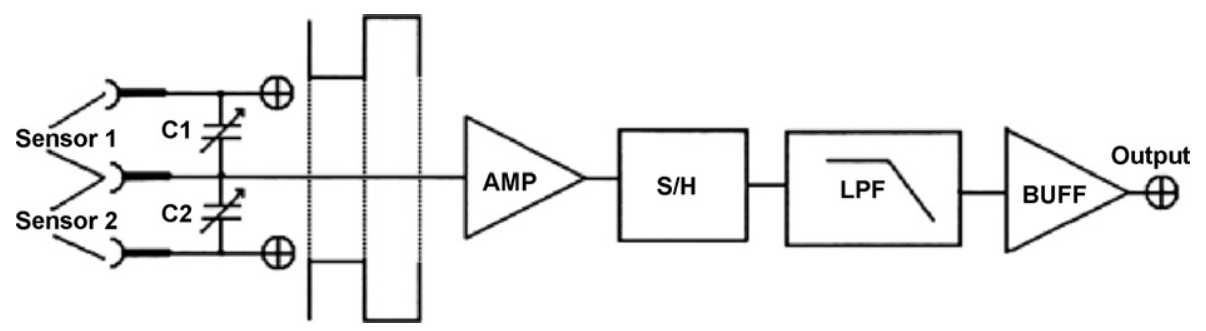

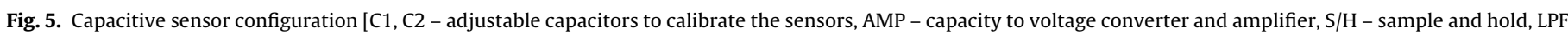
- two pole low pass filter, and BUFF - output buffer].
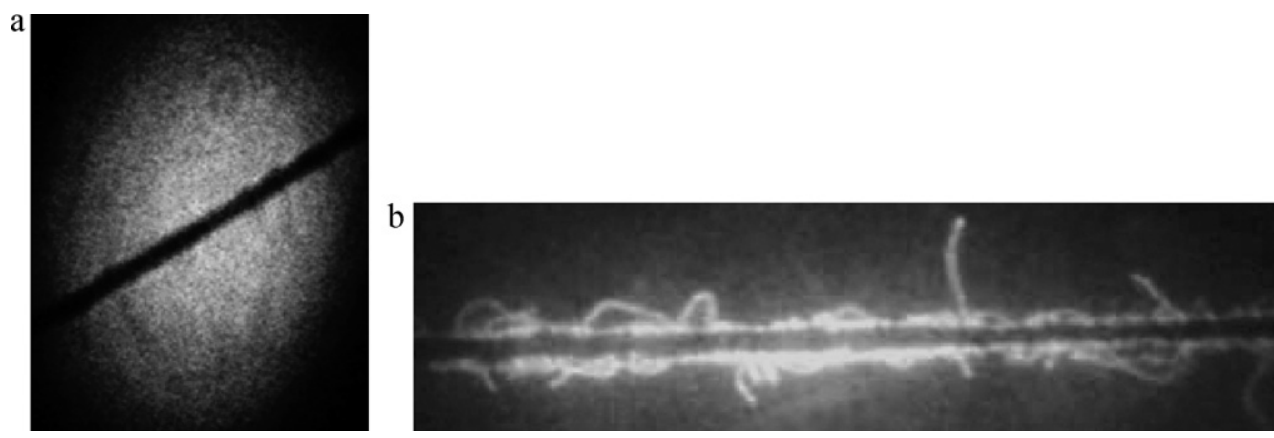

Fig. 6. Example of an image resulting from the application of a (a) low-pass spatial filter, (b) high-pass spatial filter.

for the Fourier lens, $\mathrm{L} 2$, which is 2 inches in diameter. The lenses are carefully aligned to have a common optical axis.

For the diameter measurements, basically the same optical hardware is used, with the exception that the high-pass spatial filter is replaced by a low-pass spatial filter, corresponding to a roughly $1 \mathrm{~mm}$ diameter circular aperture, i.e. the complement of the high pass spatial filter. This filter blocks all spatial frequencies above $10 \mathrm{~mm}^{-1}$,resultant to a characteristic size of $100 \mu \mathrm{m}$ or less in the object plane.

Afterwards, in order to obtain a voltage proportional to the brightness of the final image, a conditioning circuit was developed for yarn hairiness/diameter quantification. A high precision current to voltage converter based on a Burr-Brown operational amplifier OPA277P was used between the output of the photodiode and the sampling board.

Considering the hairiness measurement, the presented optical configuration overcomes the referred drawbacks of most available commercial methods. In particular it has been previously shown possible to deduce from the acquired signals a signal reference for $0 \%$ of yarn hairiness [6], permitting one to exclude to the signal due to the yarn contours. Furthermore the optical configuration is not dependent on the hairiness orientation nor on the yarn diameter, while the shape and alignment of the yarn are irrelevant as there is

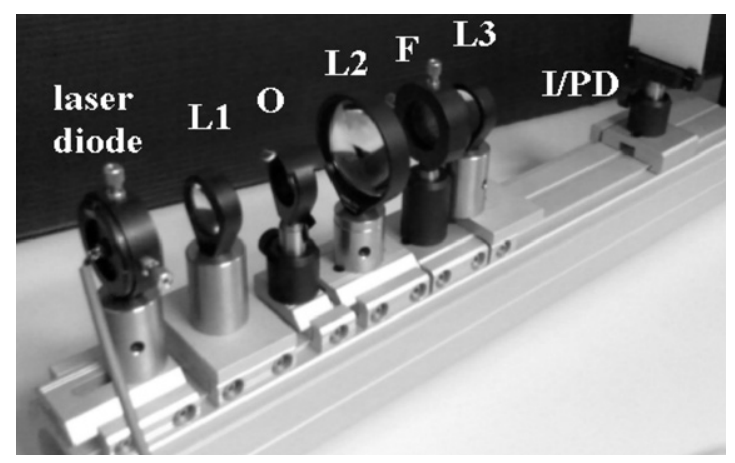

Fig. 7. Custom developed optical yarn measurement hardware for a single direction. a considerable safe area of detection in the implemented hairiness system sensor [9]. Moreover, the obtained signal is proportional to the length of hairs [6]. These characteristics are fundamental for a reliable and precise characterization of yarn hairiness. Although one might think that a full hairiness classification should require several different and simultaneous projections, the fact that the protruding hairs tend to be randomly distributed over the yarn surface, allows one to obtain a reliable estimate of the average yarn hairiness, provided a sufficient length of yarn is characterized. In a previous study [9] it was verified that the results obtained using two different simultaneous orthogonal projections give nearly identical statistical results. As a consequence, a single projection is sufficient for the correct determination of yarn hairiness [9]. The individual sample length can be adjusted to values between a few microns and $1 \mathrm{~cm}$. However, a $1 \mathrm{~mm}$ reference sample length is considered sufficient as the hairiness coefficient is determined in a centimetre range, which is ten times superior to the standard sample length used $(1 \mathrm{~cm})$. This enables the representation of the hairiness spectrogram starting at a $2 \mathrm{~mm}$ range and not on the $2 \mathrm{~cm}$ range, as in the standard sample length based equipments.

For the diameter measurement, as in the hairiness determination module, a coherent optical signal processing technique based on Fourier analysis was used to characterize yarn diameter using a singular projection [10]. This method overcomes the referred drawback of the commercial systems because the yarn hairiness is filtered by a low-pass spatial filter, increasing the measurement precision. A signal proportional to the yarn diameter is also obtained [10]. Moreover, as in hairiness measurement, it was verified that irregularities are randomly distributed over the yarn and so there is a high probability, considering the yarn length analyzed in a test, that on average, the amount of irregularities is uniformly distributed over a full rotation of the yarn [11]. A study undertaken shows that the results obtained using two orthogonal projections give nearly identical statistical results and as a consequence, a single projection is sufficient for the correct determination of diameter variation [11]. As in the hairiness module, the sample length can be easily adjusted from some microns up to $1 \mathrm{~cm}$. However, a $1 \mathrm{~mm}$ reference sample length is considered sufficient as the lowest length irregularity (nep), generally has an extension of between $1 \mathrm{~mm}$ and 

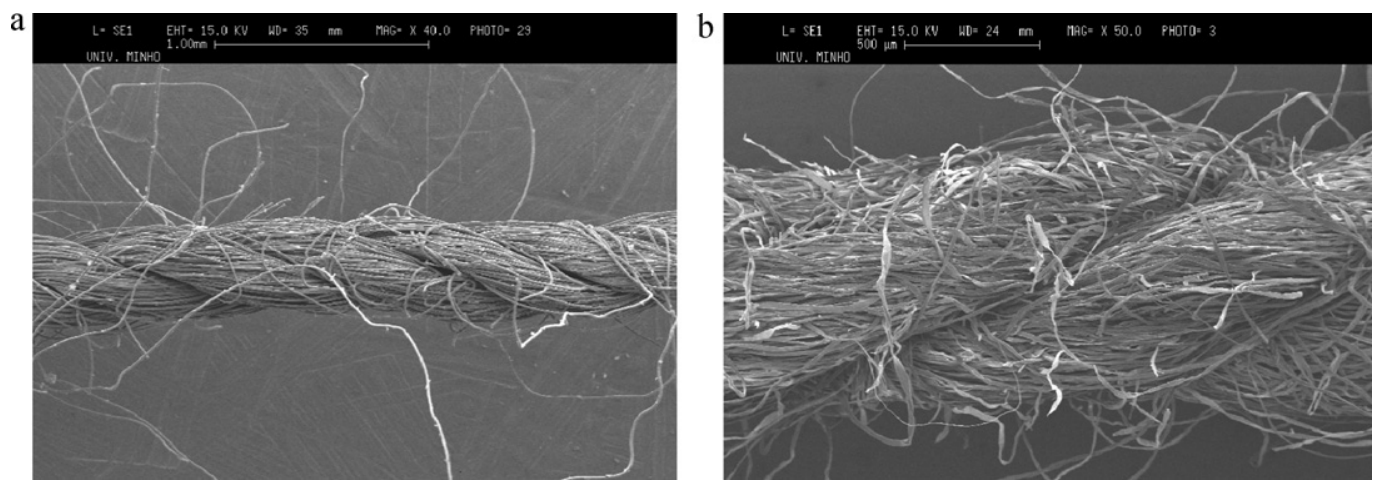

Fig. 8. Electron microscope pictures of the $59 \mathrm{~g} / \mathrm{km}$ (a) and $295 \mathrm{~g} / \mathrm{km}$ (b) yarns analyzed.

Table 1

Statistical results parameterization.

\begin{tabular}{|c|c|c|c|c|}
\hline \multirow[t]{2}{*}{ Statistical parameters (yarns) } & \multicolumn{2}{|l|}{$59 \mathrm{~g} / \mathrm{km}$} & \multicolumn{2}{|l|}{$295 \mathrm{~g} / \mathrm{km}$} \\
\hline & Capacitive sensor & Optical sensors & Capacitive sensor & Optical sensors \\
\hline Mean deviation (U) (\%) & 14.0 & 15.4 & 13.9 & 16.0 \\
\hline Coefficient of variation (CV) (\%) & 19.6 & 18.3 & 17.5 & 20.6 \\
\hline $40 \%$ Sensitivity thin places $(/ \mathrm{km})$ & 25 & 20 & 12,800 & 12,600 \\
\hline $40 \%$ Sensitivity thick places $(/ \mathrm{km})$ & 56 & 152 & 11,400 & 37,800 \\
\hline $200 \%$ Sensitivity neps (/km) & 0 & 0 & 0 & 0 \\
\hline Hairiness index $(\mathrm{H})(\mathrm{m} / \mathrm{m})$ & - & 0.3 & - & 2.2 \\
\hline Standard deviation $(\mathrm{H})(\mathrm{sH})(\mathrm{m} / \mathrm{m})$ & - & 0.1 & - & 0.6 \\
\hline
\end{tabular}

$4 \mathrm{~mm}$ (Fig. 1), which can be measured directly by the standard sample length used. In this case, as in the hairiness measurement, a laser source was used, permitting the definition of a linear saturation zone with high directionally which enables a superior system sensibility. The use of an incoherent light source would compromise all these assumptions [10].

\section{Experimental results}

Considering the optical resolution of the yarn diameter and hairiness measurements and the known relationship between yarn linear mass $(\operatorname{tex}(\mathrm{g} / \mathrm{km}))$ and diameter $(m)$ (diameter $=0.060 \sqrt{ }$ tex $)$ [12]), it is possible to correctly determine the traditional yarn parameters using only optical sensors[5,12]. In order to compare these two systems, two 100\% cotton yarns with a linear mass of 59 and $295 \mathrm{~g} / \mathrm{km}$ linear mass yarn (Fig. 8) were evaluated.

The influence of hairiness on the capacitive sensor was on average, about $3 \mathrm{E}-11 \%$ in the $59 \mathrm{~g} / \mathrm{km}$ yarn and $4 \mathrm{E}-11 \%$ in the $295 \mathrm{~g} / \mathrm{km}$ yarn, and so essentially negligible. Table 1 presents the statistical parameters obtained and Fig. 9, the signal processing results of mass variation determined directly with the capacitive sensor and inferred with the optical sensors.

As the data in Table 1 shows, regarding the main statistical parameters of mass (U\%, CV\% and irregularities) comparable results are obtained from the capacitive and optical sensors, as expected and in accordance to the previously presented relationship between yarn linear mass and diameter except for the thick places values. This can be explained by the higher resolution of the optical method. As the optical sensor only considers the yarn core in the measurements, it is more sensitive to deviations from the core mean values in more than $40 \%$ thick places. On the other hand, the fact that the capacitive sensors sample all of the yarn (core plus protruding fibres) results in these sensors being less sensitive to small deviations in the width of the core. A hairiness index $(\mathrm{H})$ of $0.3 \mathrm{~m} / \mathrm{m}$ with a standard deviation $(\mathrm{sH})$ of $0.1 \mathrm{~m} / \mathrm{m}$ is obtained for the $59 \mathrm{~g} / \mathrm{km}$ yarn and a hairiness index of $2.2 \mathrm{~m} / \mathrm{m}$ with a standard deviation of $0.6 \mathrm{~m} / \mathrm{m}$ is obtained for the $295 \mathrm{~g} / \mathrm{km}$ yarn. Further- more, as can be seen in Fig. 8a and b, a high number of similar protruding peaks (indicated with arrows) where obtained over the same wavelengths of mass variation and diameter variation characterization indicating that equal pattern distributions are present in both signals.

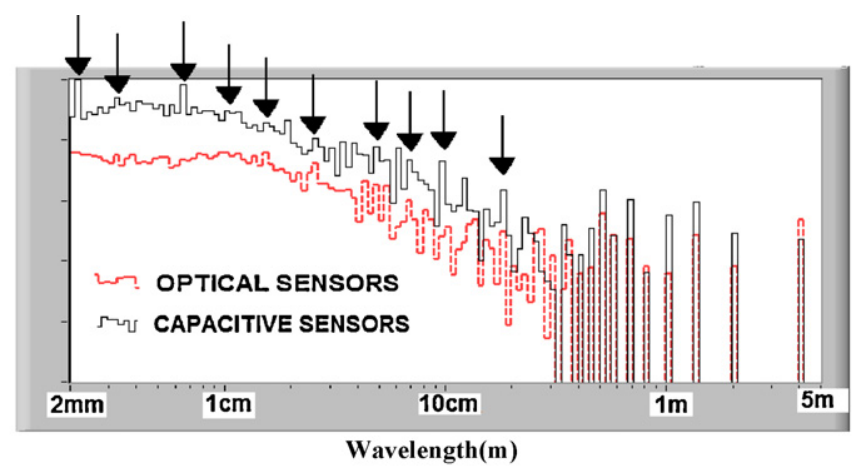

(a) $59 \mathrm{~g} / \mathrm{km}$ yarn

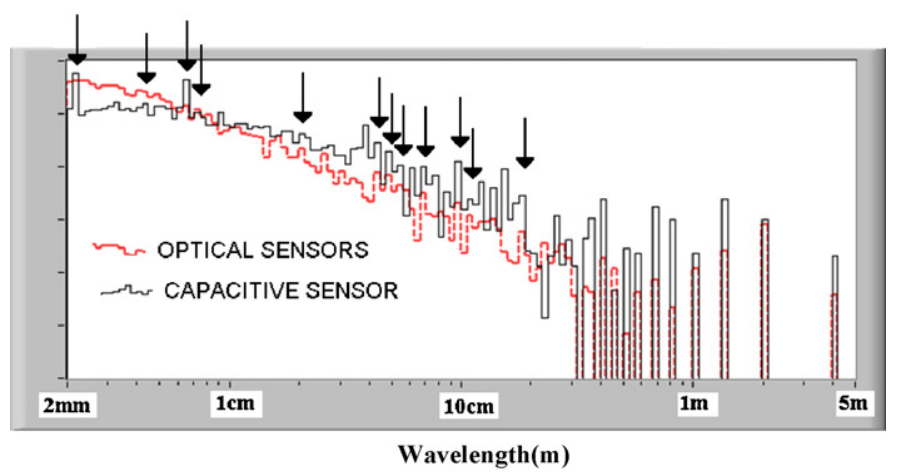

(b) $295 \mathrm{~g} / \mathrm{km}$ yarn

Fig. 9. Mass variation signal processing results based on the FFT (a). Arrows indicate the presence of similar protruding peaks in both spectrograms. 


\section{Conclusions and further work}

Considering the data obtained it is possible to state that there is a strong similarity in the parameterization data acquired using optical and capacitive sensors, implying a strong linear relationship between mass and diameter variation. These results indicate that a valid characterization of yarn mass parameters (U\%, CV\% and irregularities, between others) can be obtained using optical sensors. However, it must be noted that, the methods employed for mass measurement and diameter measurement are not absolutely equal, and that a fully accurate parameterization of mass requires a capacitive sensor, considering the different geometries and material density used in yarns. Also, in fairness, it should be remarked that optical sensors based on coherent optical techniques are inherently more sensitive to dust that might be present under industrial conditions. However, the technique presented above is based on a common path interference process and is rather insensitive to vibrations. In addition, using optical sensors it is possible to obtain very short sample lengths (less than $1 \mathrm{~mm}$, if desired), allowing one to characterize yarn with a much higher spatial resolution than can be obtained with capacitive sensor measurements.

In summary, it is possible to conclude that using only optical sensors is sufficient for an accurate yarn characterization of mass parameters, reducing systems cost, complexity and increasing efficiency of textile industry.

As already mentioned, YSQ equipment correctly characterizes yarn mass parameters and hairiness values by using capacitive and optical sensors. Moreover, although the YSQ has proven to be economically competitive in the commercial market, we want to reduce the costs using only the optical measurement. Future work will then include the development of a prototype of yarn characterization, with reduced dimension, using only optical sensors as well as the definition of quality standards for high resolution measurements (less or equal to $1 \mathrm{~mm}$ ).

\section{Acknowledgement}

The authors are grateful to the Portuguese Foundation (FCT) for providing the financial support through the scholarship (BD/19028/2004) for this project.

\section{References}

[1] V. Carvalho, M. Belsley, R. Vasconcelos, F. Soares, Automatic yarn characterization system, in: IEEE Sensors 2008, 7th IEEE Conference on Sensors, 26-29 October 2008, Lecce, Italy, 2008.
[2] V. Carvalho, Parametrização de Fio Têxtil Baseada na Análise de Massa, Msc. Thesis, Minho University, Guimarães, 2004.

[3] R. Furter, Evenness Testing in Yarn Production: Part I, The Textile Institute and Zellweger Uster AG, Manchester, 1982.

[4] J. Neves, A Irregularidade dos Fios Têxteis Sua Origem, Medição e Análise, Oporto, 1968.

[5] B. Goswami, J. Martindale, F. Scardino, Textile Yarns: Technology Structure and Applications, 2nd edition, John Wiley \& Sons, New York, 1977.

[6] V. Carvalho, P. Cardoso, M. Belsley, R. Vasconcelos, F. Soares, Yarn hairiness parameterization using a coherent signal processing technique, Sensors and Actuators A: Physical 145 (1) (2008) 217-224.

[7] V. Carvalho, P. Cardoso, M. Belsley, R. Vasconcelos, F.O. Soares, Yarn diameter measurements using coherent optical signal processing, IEEE Sensors Journal 8 (11) (2008) 1785-1793.

[8] Barella, Yarn hairiness, Textile Progress 13 (1981) 1.

[9] V. Carvalho, P. Cardoso, M. Belsley, R. Vasconcelos, F.O. Soares, Yarn Hairiness characterization using two orthogonal directions, IEEE Transactions on Instrumentation and Measurement 58 (3) (2009) 594-601.

[10] V. Carvalho, P. Cardoso, M. Belsley, R. Vasconcelos, F. Soares, Yarn diameter measurements using coherent optical signal processing, IEEE Sensors Journal 8 (11) (2008) 1785-1793.

[11] V. Carvalho, P. Cardoso, M. Belsley, R. Vasconcelos, F. Soares, Yarn diameter characterization using two orthogonal directions, Journal Experimental Techniques (2010), doi:10.1111/j.1747-1567.2010.00632.x (published online in 12th, April).

[12] V Carvalho, M. Belsley, R. Vasconcelos, F.O. Soares, Yarn diameter and linear mass correlation, Journal of Non-Destructive Evaluation 28 (2) (2009) 49-54.

\section{Biographies}

V. Carvalho received his degree in industrial electronics engineering in the option of telecommunications and industrial informatics, in 2002 and MSc in industrial electronics, in the option of automation and robotics in 2004, both from Minho University, Portugal. He concluded his $\mathrm{PhD}$ in industrial electronics in 2008 , covering the subject presented here. Actually he is working as assistant professor at the Polytechnic Institute of Cávado and Ave (IPCA) and Portuguese Catholics University (UCP). His main fields of interest are data acquisition and signal processing.

M. Belsley obtained his PhD degree in physics from the University of Colorado at Boulder in 1986. He then worked at the California State University in Long Beach, Oxford University and the University of Oregon before coming to Minho University in Braga Portugal where he has lectured as an associate professor of physics since 1992. His main fields of interest are laser spectroscopy and nonlinear optics.

R. Vasconcelos received her degree in textile engineering, in 1984 at Minho University, Portugal. In 1993 she obtained her PhD in engineering-textile technology and chemistry on the speciality of textile technology, in Minho University. Since 2005 she is working as associate professor, in the Textile Engineering Department of Minho University. Her fields of interest are textile processes and industrial automation.

F. Soares received her degree in chemical engineering, in 1986 at Porto University, Portugal. In 1997 she obtained her PhD in chemical engineering in Porto University. Since 2007 she is working as associate professor, in the Industrial Electronics Department of Minho University. Her fields of interest are process modelling and control and process automation. 